HNO 2020 • 68:393-394

https://doi.org/10.1007/s00106-020-00832-1

Online publiziert: 5. März 2020

(c) Springer Medizin Verlag GmbH, ein Teil von Springer Nature 2020

\section{K.-Wolfgang Delank}

HNO-Klinik, Klinikum der Stadt Ludwigshafen, Ludwigshafen, Deutschland

\title{
Festschrift für Thomas Deitmer
}

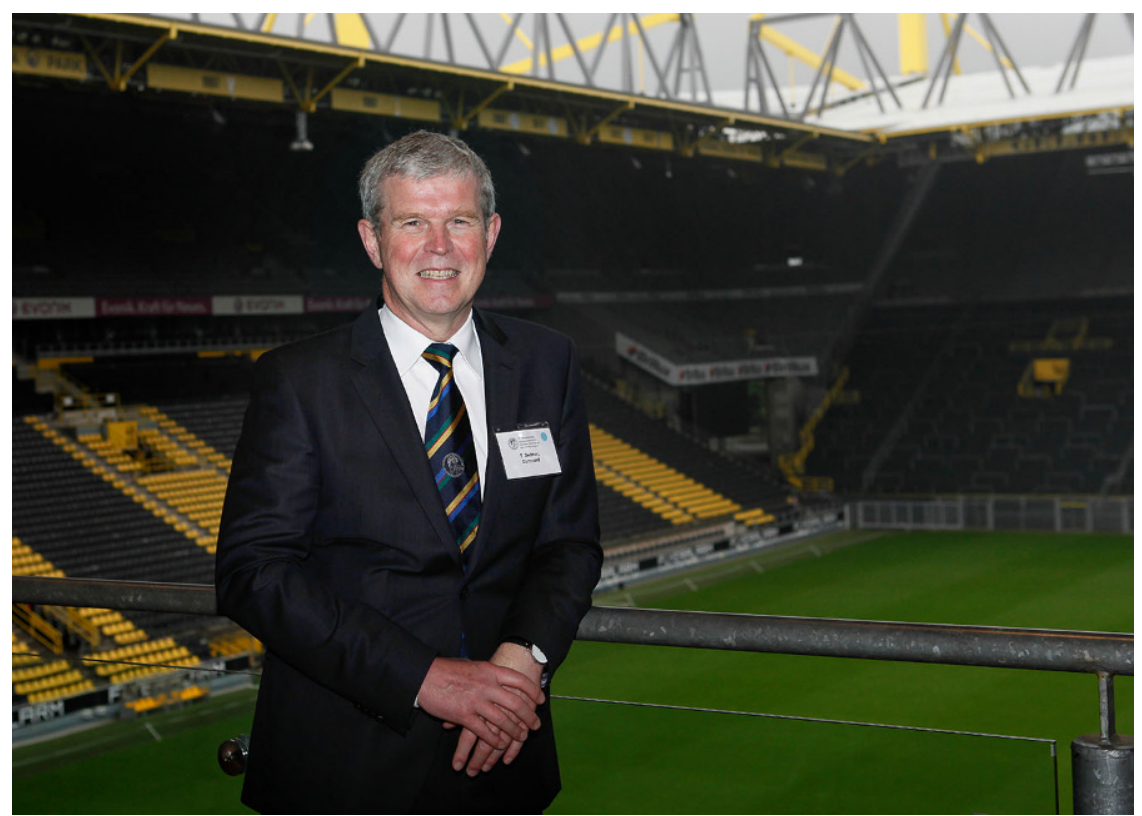

Abb. 1 ॥ Empfang des Präsidenten 2014: T. Deitmer mit Blick in das Stadion des BVB Dortmund (ODGHNO KHC)

Sehr geehrte, liebe Kolleginnen und Kollegen,

beim Blick auf die Titelseite und beim Blättern wird es Ihnen sofort auffallen: Sie halten eine Ausgabe der HNO in den Händen, die einer außergewöhnlich angesehenen Persönlichkeit der HNOCommunity aus einem besonderen Anlass gewidmet ist. Prof. Dr. med. Thomas Deitmer hat am 01.08.2019 nach 23 Jahren seine Tätigkeit als Direktor der HNO-Klinik am Städtischen Klinikum Dortmund offiziell beendet, um künftig als hauptamtlicher Generalsekretär der Deutschen Gesellschaft für Hals-NasenOhren-Heilkunde, Kopf- und Hals-Chirurgie (DGHNO) mit Rat und Tat zur Seite stehen zu können. Im Rahmen eines festlichen Symposiums mit zahlreichen Gästen übergab er am 07.09.2019 die „Stafette" an Prof. Dr. med. Claus Wittekindt, der zuletzt die HNO-Universitätsklinik in Gießen kommissarisch leitete.

Prof. Dr. med. Thomas Deitmer, geboren am 18. Juni 1954 im sauerländischen Hemer, erhielt nach Medizinstudium und Promotion an der Westfälischen Wilhelms-Universität (WWU) ab 1980 seine HNO-ärztliche Weiterbildung und seine wissenschaftliche Prägung an der dortigen Universitäts-HNO Klinik bei Prof. Dr. med. Dr. h.c. Harald Feldmann und dessen Nachfolger, Prof. Dr. Wolfgang Stoll. Bereits kurze Zeit nach dem Erwerb der Facharztqualifikation wurde er zum Oberarzt und 1991 zum leitenden Oberarzt berufen. Er besitzt die Zusatzbezeichnungen plastische Operationen und spezielle HNO-Chirurgie. Schon früh beschäftigte er sich mit einer breiten Palette klinisch-wissenschaftlicher Themen zu denen die Epidemiologie des Larynxkarzinoms bei Frauen sowie der asbestin- duzierte Kehlkopfkrebs zählten. Parallel fokussierte sich Thomas Deitmer auf die Erforschung der in den 1980er-Jahren noch weitgehend unbekannten mukoziliaren Transportmechanismen der Nase, die dann auch Gegenstand seiner Habilitationsschrift 1987 wurden. Im Jahr 1989 publizierte er im Karger-Verlag die international mit großem Interesse wahrgenommene Monographie Physiology and Pathology of the Mucociliary System. Aufgrund seiner beachtenswerten Leistungen in Forschung und Lehre wurde er 1993 von der WWU zum Professor ernannt. Sein wissenschaftliches Werk umfasst über 90 wissenschaftliche Publikationen, Übersichtsreferate und Buchbeiträge. Seit Jahren ist Thomas Deitmer regelmäßig als eingeladener Referent auf großen, nationalen und internationalen Kongressen anzutreffen.

Während seiner Münsteraner Zeit pflegte Thomas Deitmer einen besonders intensiven fachlichen Austausch mit der dortigen Universitäts-Kinderklinik, in der seine charmante Ehegattin MarieLuise als Pädiaterin beschäftigt war und ihm pädiatrische HNO-Fälle „auf dem kurzen Dienstweg“ vorstellte. $\mathrm{Zu}$ den besonders komplizierten Fällen zählten nicht wenige Kinder mit angeborenen oder erworbenen Kehlkopfdysplasien und Trachealstenosen. Möglicherweise war es die Verknüpfung von echter therapeutischer Herausforderung, Empathie für die kleinen Patienten und ehelichem Zuspruch, die Thomas Deitmer zu einer gezielten Weiterentwicklung seiner Qualifikation in der bekanntermaßen komplizierten Tracheal- und Larynxchirurgie bei Kindern animierte. Die erforderlichen Spezialkenntnisse eignete er sich u. a. bei Professor Robin T. Cotton in Cincinnati/OH, USA, sowie bei Hospitationen im Great Ormond Street 
Hospital for Children in London, GB, an.

Nach einer kurzen Episode als Chefarzt der HNO-Klinik in Augsburg übernahm Thomas Deitmer am 01.08.1996 als Nachfolger von Prof. Dr. med. Rose die ärztliche Leitung der renommierten HNO-Klinik am Städtischen Klinikum in Dortmund. In den folgenden 23 Jahren profitierte die Klinik nicht nur von seiner außergewöhnlich breiten Fachkompetenz, sondern auch von seiner klugen, von Teamgeist und Fairness geprägten Personalführung. Die niedergelassene HNO-Kollegenschaft schätzt ihn als absolut verlässlichen, höchst kompetenten Kooperationspartner und guten Ratgeber. Kurz: Die Dortmunder HNO-Klinik gilt als medizinischer Leuchtturm, der weit über das nördliche Ruhrgebiet hinaus wahrgenommen wird.

\section{》) Auf seine Initiative erfolgte 2014 die Gründung der PädHNO}

So war Professor Deitmer 11 Jahre lang Vorsitzender der Vereinigung der HNOChefärzte und Ordinarien sowie ab 2006 Mitglied des Präsidiums der DGHNO. Als deren Präsident richtete er 2014 die 85. Jahresversammlung in Dortmund aus. Gern erinnern sich viele von uns an den sehr harmonischen und wissenschaftlich attraktiven Kongress in den Westfalenhallen, der unter dem Motto „HNO für jedes Lebensalter“ genügend Raum ließ für die pädiatrischen Aspekte unseres Fachs, die Thomas Deitmer persönlich bis heute sehr wichtig sind. Auf seine Initiative erfolgte 2014 die Gründung der Arbeitsgemeinschaft pädiatrische HNO-Heilkunde (PädHNO), der er als Vorsitzender wichtige Impulse gab und deren Aktivitäten und inhaltliche Ausrichtung er nach wie vor als Mitglied des Vorstands sehr aufmerksam begleitet. International engagiert er sich in der European Society of Pediatric Otolaryngology (ESPO) als Delegierter der DGHNO.

Nicht erst seit seiner Präsidentschaft ist Thomas Deitmer für sein berufsund standespolitisches Engagement bekannt. Mit Diplomatie, Pragmatismus und Beharrlichkeit setzt er sich seit Jahren für die Belange unserer Fachgesellschaft ein, pflegt wertvolle Kontakte zu den Nachbardisziplinen und führt erfolgreich Verhandlungsgespräche in politischen Gremien in Berlin, etwa im Gemeinsamen Bundesausschuss (GBA) und in der Bundesärztekammer. Komplexe Aufgaben, wie die Supervision der fachspezifischen Aspekte der DRG und die kritische Begleitung der Novellierung der Gebührenordnung für Ärzte (GOÄ) sind bei ihm bestens aufgehoben. Für die langjährige, erfolgreiche Leitung der GOÄ-Kommission wurde ihm 2017 die Verdienstmedaille der DGHNO verliehen.

Thomas Deitmer ist der Entschluss, die Leitung seiner angesehenen Klinik in Dortmund und die klinische Tätigkeit aufzugeben, nicht leicht gefallen. Wer ihn genau kennt, weiß, dass sein Herz beruflich v. a. für die ihm anvertrauten Patienten, seine Klinik und seine Dortmunder HNO-Crew schlägt. Die vorliegende Sonderausgabe der HNO soll ihm den Abschied ein wenig erleichtern und zudem herzlichen Dank für seine Bereitschaft, dem Ruf aus der Bonner Friedrich-Wilhelm-Straße zu folgen, zum Ausdruck bringen. Ihn als hauptamtlichen Generalsekretär gewonnen zu haben, dürfte für die DGHNO und unser Fach ein echter Glückfall sein.

Dir, lieber Thom, bleibt zu wünschen, dass Dir die neue Aufgabe nicht nur viel Freude und Zufriedenheit bereiten wird, sondern auch genügend Zeit für regelmäßige Reisen nach Kiel („Enkelhausen“) und das Coaching Deiner geliebten, sportlichen Enkelkinder lässt.

\section{K.-Wolfgang Delank}

\section{Korrespondenzadresse}

Prof. Dr. med. K.-Wolfgang Delank HNO-Klinik, Klinikum der Stadt Ludwigshafen Bremserstr. 79, 67063 Ludwigshafen, Deutschland delankw@klilu.de

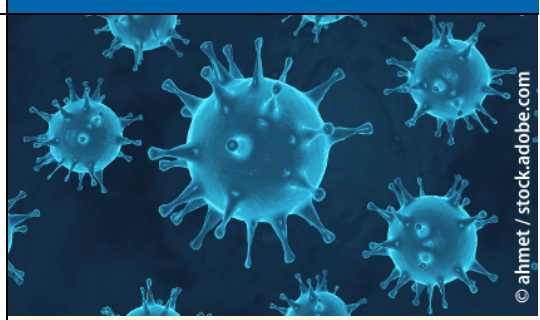

\section{Aktuelle Entwicklung zu COVID-19 bei Springer Nature und Springer Medizin}

Springer Nature und Springer Medizin unterstützen die globale Reaktion auf die COVID-19-Pandemie, indem ein schneller und direkter $\mathrm{Zu}$ gang zu den neuesten verfügbaren Forschungsergebnissen und Daten ermöglicht wird.

Auf der Homepage SpringerMedizin.de finden Sie ein immer aktuelles Dossier mit Beiträgen, Forschungsarbeiten und Ergebnissen zu SARS-CoV-2 sowie relevanten Links.

Darin z.B. auch die kürzlich publizierte

Empfehlung von DIVI, DGIIN, DGAI und DGP zur Intensivtherapie von Patienten mit COVID-19.

Springer Nature arbeitet mit globalen Organisationen zusammen, und verlinkt über SpringerNature.com/de auf eine eigene Landingpage mit einer Vielzahl an Information sowie freiem Zugriff auf die COVID-19-Contentplattformen von Nature Research, BioMed Central (BMC) und Springer.

Das Dossier zu Coronavirus / Covid-19 von Springer Medizin finden Sie hier: www.springermedizin.de/covid-19

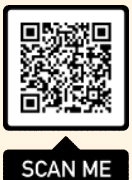

